\title{
2-tuple Linguistic Fuzzy Stochastic MAGDM Method Based on Prospect Theory and Cloud Model
}

\author{
Li Xiaorui \\ School of Business \\ Zhengzhou university of Industrial Technology \\ Xinzheng, 451100, P. R. China \\ Xiaorui_Lii@163.com
}

\begin{abstract}
This paper presents a new approach to solving the fuzzy stochastic multi attributes group decision-making (MAGDM) problem where the attributes values take 2-tuple linguistic form. First, a 2-tuple hybrid ordered weighted geometric (THOWG) operator is developed and all the individual preference values are aggregated into the comprehensive preference values. Then, by applying generating cloud method, a comprehensive preference value is converted into a normal cloud. Consequently, the cloud prospect decision matrix is constructed by defining a cloud distance, cloud possibility degree and cloud prospect value function. Furthermore, we establish a programming model which satisfies maximum integrated cloud prospect value, attain the attributes weights, and consequently list the order of alternatives. Finally, an example is illustrated to verify the developed approach.
\end{abstract}

Keywords-Prospect theory; multi-attributes group decisionmaking; Cloud model; 2-tuple linguistic

\section{INTRODUCTION}

Multiple attributes group decision-making (MAGDM) is an important research topic, which determines the ranking order of the alternatives to help decision makers to make their decisions. In reality, the decision-making information is usually uncertain or fuzzy due to the complexity of object factors and the recognizing limitations of decision makers. Moreover, in many situations, the attributes values of decision-making information take the form of 2-tuples linguistic variables because of time pressure, lack of knowledge, and people's limited expertise related with problem domain. Herrera and Martinez proposed a 2-tuple linguistic model to represent the assessment information which can effectively avoid information loss[1-2]. Recently, many researchers paid much attention to 2-tuple linguistic MAGDM problems[3-8].

The 2-tuple linguistic model can describe the decisionmaking information, whereas it does not seem perfect and accurate to deal with information in terms of fuzziness and randomness. In this respect, the Cloud model can well overcome this weakness and make decision processes more realistic as it characterizes information by using fuzzy set theory and probability statistics at the same time. The Cloud model proposed by Professor Deyi Li, which is based on the traditional fuzzy set theory and probability statistics, not only well characterizes the concept of uncertainty in the natural language, but also reflects the intrinsic connection between randomness and fuzziness[9]. Owing to the advantage of tackling with vague and random information, many methods on Cloud model have been put forward and used to solve MAGDM problems[10-13]. Hence, it is necessary to incorporate Cloud model into the 2-tuple linguistic to describe the decision-making information.

However, these researches mentioned above generally assumed that decision makers were totally rational and fell into the expected utility theory framework. Nevertheless, the expected utility theory has some unexplained phenomena such as Allias paradox and Ellsberg paradox. Noting the limitations of the expected utility theory, Kahneman and Tversky proposed Prospect theory[14]. The decision-making method based on Prospect theory has recently become a research hotspot because it is more in line with people's actual decisionmaking behavior[15-17]. To the best of authors' knowledge, however, the study of 2-tuple linguistic fuzzy stochastic MAGDM problems based on Cloud model and Prospect theory at present has not been reported in the existing literature.

The paper aims to develop a new method for studying 2tuple linguistic fuzzy stochastic MAGDM problems via Cloud model and Prospect theory. A MAGDM method largely consists of four phases: (1) Construct decision problem and describe information; (2) aggregate the input arguments; (3) determine its weights; and (4) multiply these arguments and its weights, and then aggregate all the weighted arguments.

As for the second phase, there are many methods for aggregating the information[1,3,5-7,18-20]. In these aggregating operators, 2-tuple weighted geometric (TWG) operator and 2-tuple ordered weighted geometric (TOWG) operator are the most popular due to their easy calculation, good reliability and high resolution. However, TWG operator just considers individual importance, and it neglects the importance of ordered position. On the other hand, TOWG operator only takes into account the importance of relative position while it ignores the individual importance. Hence, we propose a 2-tuple hybrid ordered weighted geometric (THOWG) operator to consider not only the individual importance but also the importance of relative position. In addition, some properties of the THOWG operator are analyzed. In particular, the THOWG operator can respectively degenerate to TWG operator and TOWG operator under the given conditions. 
Furthermore, by using THOWG operator, we derive comprehensive preference values and consequently, a comprehensive preference value can be converted into a normal cloud by applying generating cloud method. Based on Prospect theory and Cloud model, we define a cloud distance, cloud possibility degree and cloud prospect value function. According to these definitions, we construct the cloud prospect decision-making matrix which selects all other alternatives as the reference point. Considering the risk attitudes of decision makers, a programming optimization model is constructed to calculate the attributes weights by applying the method of maximizing the integrated cloud prospect value. Finally, based on the cloud prospect decision-making matrix and attributes weighting model, the integrated cloud prospect value is obtained, which provides a scale for ordering alternatives. In addition, an example is provided to show the validity of the approach.

The rest of this paper is organized as follows. Section 2 introduces the fundamental conceptions of 2-tuple linguistic, Prospect theory and Cloud model. Section 3 develops a new aggregation (THOWG) operator and provides a method by which a 2-tuple linguistic is converted into a corresponding normal cloud. We further define the cloud distance, cloud possibility degree and cloud prospect value function. Section 4 proposes a 2-tuple linguistic fuzzy stochastic MAGDM approach based on Prospect theory and Cloud model. Section 5 presents an example to verify our method and Section 6 draws conclusions.

\section{PRELIMINARIES}

This section introduces the fundamental conceptions of 2tuple linguistic, Cloud model and Prospect theory.

\section{A. 2-tuple linguistic}

First, Herrera and Martinez developed a 2-tuple fuzzy linguistic representation model based on the concept of symbolic translation[1-2]. It is used for representing the linguistic assessment information by means of a 2-tuple $\left(s_{i}, a_{i}\right)$, where $s_{i}$ is a linguistic label from predefined linguistic term set $\mathrm{S}$ and $a_{i}$ is the value of symbolic translation, and $a_{i} \in[-0.5,0.5)$.

Definition 1 Let $S=\left\{s_{0}, s_{1}, s_{2}, \cdots, s_{t}\right\}$ be a finite and totally ordered discrete linguistic term set with odd cardinality, where $s_{i}$ represents a possible value for a linguistic variable. $\beta \in[0, t]$ is a number value representing the aggregation result of linguistic symbol. Then the function $\Delta$ used to obtain the 2-tuple linguistic information equivalent to $b$ is defined as:

$$
\Delta:[0, Q] \rightarrow S \times[-0.5,0.5), \beta \rightarrow \Delta(\beta)=\left(s_{i}, \alpha\right),
$$

where $i=\operatorname{round}(\beta), \alpha=\beta-i, \alpha \in[-0.5,0.5)$, round $(\cdot)$ is the usual round operation, $s_{i}$ has the closest index label to $\beta$ and $\alpha$ is the value of the symbolic translation.
Definition 2 Let $S=\left\{s_{0}, s_{1}, s_{2}, \cdots, s_{t}\right\}$ be a linguistic term set and $\left(s_{i}, \alpha\right)$ a linguistic 2-tuple. There is always a function $\Delta^{-1}$ such that from a 2 -tuple it returns its equivalent numerical value $\beta \in[0, t] \subset R$, which is

$$
\Delta^{-1}: S \times[-0.5,0.5) \rightarrow[0, t], \Delta^{-1}\left(s_{i}, \alpha\right)=i+\alpha=\beta .
$$

From Definitions 1 and 2, we can conclude that the conversion of a linguistic term into a linguistic 2-tuple consists of adding a value 0 as symbolic translation:

$$
\Delta\left(s_{i}\right)=\left(s_{i}, 0\right) \text {. }
$$

Definition 3 Let $\left(s_{k}, \alpha_{k}\right)$ and $\left(s_{l}, \alpha_{l}\right)$ be two 2-tuples, they should have the following properties:

(1) If $k<l$ then $\left(s_{k}, \alpha_{k}\right)$ is smaller than $\left(s_{l}, \alpha_{l}\right)$, denoted by $\left(s_{k}, \alpha_{k}\right)<\left(s_{l}, \alpha_{l}\right)$;

(2) If $k=l$ then (a) if $\alpha_{k}=\alpha_{l}$, then $\left(s_{k}, \alpha_{k}\right)$ $=\left(s_{l}, \alpha_{l}\right)$, representing the same information; (b) if $\alpha_{k}<\alpha_{l}$, then $\left(s_{k}, \alpha_{k}\right)<\left(s_{l}, \alpha_{l}\right)$; (c) if $\alpha_{k}>\alpha_{l}$, then $\left(s_{k}, \alpha_{k}\right)>\left(s_{l}, \alpha_{l}\right)$.

\section{B. Cloud model}

The cloud theory is a model that contains the transferring procedure of uncertainty between quality concept and quantity data representation by using natural language, which was proposed by Professor Deyi Li based on the traditional fuzzy set theory and probability statistics[9].

Definition 4 Suppose $U$ is a quantitative domain expressed by precise values, and $\mathrm{C}$ is a qualitative concept on the domain. If the quantitative value $x \in U$, and $\mathrm{x}$ is a random realization of $\mathrm{C}$, whose membership $\mu(x) \in[0,1]$ is a random number with stable tendency:

$$
\mu: U \rightarrow[0,1], \forall x \in U, x \rightarrow \mu(x) .
$$

Then, the distribution of $x$ in the domain is called as cloud, and each $x$ is called as droplet.

Cloud is made up of many cloud droplets, and a single cloud droplet is a specific realization of the qualitative value in number. The three number features of cloud are expectation $E x$, entropy $E n$ and hyper entropy $H e$. Here, Ex determines the center of the cloud, En determines the range of the cloud and $\mathrm{He}$ determines the dispersive degree of cloud droplets.

The $3 E n$ rules of cloud refer to that the total contribution of all elements on the domain $\mathrm{U}$ to the qualitative concept $\mathrm{C}$ is 1.That is, $99.7 \%$ of the cloud droplets will fall into the range $(E x-3 E n, E x+3 E n)$. Normal cloud is the most common to express the linguistic values. Figure 1 shows the cloud (50, $3.93,0.1$ ). 


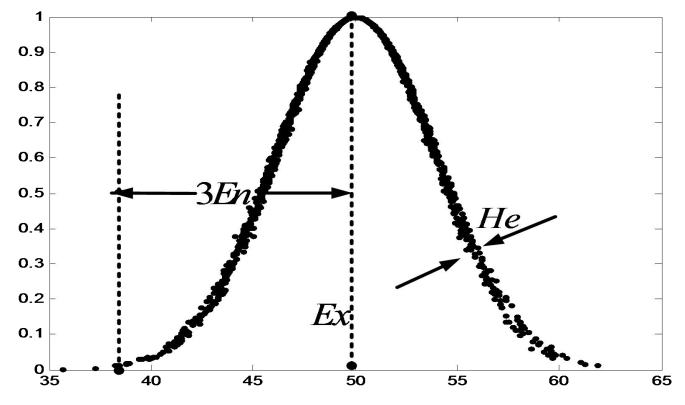

Figure 1.cloud (50, 3.93, 0.1)

\section{Prospect theory}

Prospect theory was initially proposed by Kahneman and Tversky[14]. The theory considers that the decision results are associated with the subjective standard of decision maker under uncertain conditions, and different decision makers may have different options on the same issue. Prospect theory is codetermined by the value function and the probability weight function.

$$
V=\sum_{i=1}^{n} \omega\left(p_{i}\right) v\left(\Delta x_{i}\right)
$$

where $\mathrm{V}$ is the prospect value, and $\omega\left(p_{i}\right)$ is the probability weight function which is the monotone increasing function of the probability assessment, and $v\left(\Delta x_{i}\right)$ is the value function coming from the subjective feeling of decision maker. $v\left(\Delta x_{i}\right)$ is proposed by Tversky and Kahneman[21]:

$$
v(\Delta x)= \begin{cases}(\Delta x)^{\alpha}, & (\Delta x \geq 0), \\ -\theta(-\Delta x)^{\beta}, & (\Delta x<0),\end{cases}
$$

where $\Delta x$ is used to measure the value deviation from a certain reference point, which is the gains or the losses of the surface value. $\alpha$ and $\beta$ are parameters related to gains and losses, respectively. The parameter $\theta$ represents a characteristic of being steeper for losses than for gains and $\theta>1$ shows the loss aversion.

The probability weight function proposed by Tversky and Kahneman is shown as follows[21]:

$$
\omega^{+}(p)=\frac{p^{\gamma}}{\left(p^{\gamma}+(1-p)^{\gamma}\right)^{1 / \gamma}}, \omega^{-}(p)=\frac{p^{\delta}}{\left(p^{\delta}+(1-p)^{\delta}\right)^{1 / \delta}},
$$

where $\omega^{+}(p)$ and $\omega^{-}(p)$ are respectively the nonlinear weight function of the gains and the losses, and $\gamma$ is the risk gain attitude coefficient, and $\delta$ is the risk loss attitude coefficient.

\section{THE CLOUD MODEL ALGORITHM AND CLOUD PROSPECT VALUE FUNCTION}

This section provides a new 2-tuple linguistic aggregation operator, and presents a method that a 2-tuple linguistic is converted into a corresponding normal cloud. In addition, this section defines a cloud distance, cloud possibility degree and cloud prospect value function.

\section{A. Aggregation operators with 2-tuple linguistic information and generating cloud method}

1) Aggregation operators with 2-tuple linguistic information.

Definition 5[18] Jiang \& Fan let $\left\{\left(s_{1}, a_{1}\right),\left(s_{2}, a_{2}\right)\right.$ $\left., \cdots,\left(s_{n}, a_{n}\right)\right\}$ be a set of 2-tuple, and the weighting vector of 2tuple $\left(s_{j}, a_{j}\right)$ is $\omega=\left(\omega_{1}, \omega_{2}, \cdots, \omega_{n}\right)^{T}$ such that $\omega_{j}>0$, $\sum_{j=1}^{n} \omega_{j}=1$. The 2-tuple weighted geometric (TWG) operator is

$$
(\tilde{s}, \tilde{\alpha})=\Delta\left(\prod_{j=1}^{n}\left(\Delta^{-1}\left(s_{j}, \alpha_{j}\right)\right)^{\omega_{j}}\right), \quad \tilde{s} \in S, \tilde{\alpha} \in[-0.5,0.5) .
$$

Definition 6[18] Jiang \& Fan let $\left\{\left(s_{1}, a_{1}\right),\left(s_{2}, a_{2}\right)\right.$ $\left., \cdots,\left(s_{n}, a_{n}\right)\right\}$ be a set of 2-tuples, a 2-tuple ordered weighted geometric operator of $\mathrm{n}$ dimension is a mapping TOWG $: R^{n} \rightarrow R$ that has an associated vector $\omega=\left(\omega_{1}, \omega_{2}, \cdots, \omega_{n}\right)^{T}$ such that $w_{j}>0$ and $\sum_{j=1}^{n} \omega_{j}=1$, moreover,

$$
(\bar{s}, \bar{\alpha})=\Delta\left(\prod_{j=1}^{n}\left(\Delta^{-1}\left(s_{\pi(j)}, \alpha_{\pi(j)}\right)\right)^{\omega_{j}}\right), \quad \bar{s} \in S, \bar{\alpha} \in[-0.5,0.5),
$$

where $(\pi(1), \pi(2), \cdots, \pi(n))$ is a permutation of $(1,2, \cdots, n)$ such that $\left(s_{\pi(j-1)}, \alpha_{\pi(j-1)}\right) \geq\left(s_{\pi(j)}, \alpha_{\pi(j)}\right)$ for all $j=1,2, \cdots, \mathrm{n}$.

From definition 5 and 6, we find that TWG operator only considers individual importance, whereas it neglects the importance of ordered position. On the other hand, TOWG operator just focuses on the importance of relative position while it ignores the individual importance.

Therefore, this paper presents a new 2-tuple aggregation operator to overcome these limitations.

Definition 7 Let $\left\{\left(s_{1}, a_{1}\right),\left(s_{2}, a_{2}\right), \cdots,\left(s_{n}, a_{n}\right)\right\}$ be a set of 2-tuples, the 2-tuple hybrid ordered weighted geometric (THOWG) operator of $\mathrm{n}$ dimension is a mapping THOWG: $R^{n} \rightarrow R$ that 
has an associated vector $\omega=\left(\omega_{1}, \omega_{2}, \cdots, \omega_{n}\right)^{T}$ such that $\omega_{j}>0$ and $\sum_{j=1}^{n} \omega_{j}=1$. Furthermore,

$$
(\hat{s}, \hat{\alpha})=\Delta\left(\prod_{j=1}^{n}\left(\Delta^{-1}\left(\widehat{s}_{\pi(j)}, \widehat{\alpha}_{\pi(j)}\right)\right)^{\omega_{j}}\right), \quad \hat{s} \in S, \hat{\alpha} \in[-0.5 .0 .5),
$$

where $(\pi(1), \pi(2), \cdots, \pi(n))$ is a permutation of $(1,2, \cdots, n)$, such that $\left(\widehat{s}_{\pi(j-1)}, \hat{\alpha}_{\pi(j-1)}\right) \geq\left(\widehat{s}_{\pi(j)}, \hat{\alpha}_{\pi(j)}\right)=$ $\Delta\left(\Delta^{-1}\left(s_{j}, \alpha_{j}\right) / n w_{j}\right)$ for all $j=2, \cdots, n$, here $w=$ $\left(w_{1}, w_{2}, \cdots, w_{n}\right)^{T}$ is the weighting vector of 2-tuple $\left(s_{j}, a_{j}\right)$ and $w_{j}>0, \sum_{j=1}^{n} w_{j}=1$.

Proposition 1. If $w=(1 / n, 1 / n, \cdots, 1 / n)^{T}$, THOWG operator degenerates into TOWG operator.

Proof. If $w=(1 / n, 1 / n, \cdots, 1 / n)^{T}$, then $\Delta\left(\Delta^{-1}\left(s_{j}, \alpha_{j}\right) / n w_{j}\right)$ $=\left(s_{j}, \alpha_{j}\right)$.

Thus,

$$
\begin{aligned}
& \operatorname{THOWG}\left(\left(s_{1}, \alpha_{1}\right),\left(s_{2}, \alpha_{2}\right), \cdots,\left(s_{n}, \alpha_{n}\right)\right) \\
& =\operatorname{TOWG}\left(\left(s_{1}, \alpha_{1}\right),\left(s_{2}, \alpha_{2}\right), \cdots,\left(s_{n}, \alpha_{n}\right)\right) .
\end{aligned}
$$

Proposition 2. If $w=(1 / n, 1 / n, \cdots, 1 / n)^{T}$ and $\left(s_{j-1}, \alpha_{j-1}\right) \geq$ $\left(s_{j}, \alpha_{j}\right)$, then THOWG operator degenerates into TWG operator.

Proof. According to Proposition 1, we know that THOWG operator degenerates into TOWG operator as $w=(1 / n, 1 / n, \cdots, 1 / n)^{T}$. In addition, as $\left(s_{j-1}, \alpha_{j-1}\right) \geq\left(s_{j}, \alpha_{j}\right)$, the corresponding weight of $\left(s_{j}, \alpha_{j}\right)$ is $\omega_{j}$. Therefore, as $w=(1 / n, 1 / n, \cdots, 1 / n)^{T}$ and $\left(s_{j-1}, \alpha_{j-1}\right) \geq\left(s_{j}, \alpha_{j}\right)$, we have

$$
\begin{aligned}
& \operatorname{THOWG}\left(\left(s_{1}, \alpha_{1}\right),\left(s_{2}, \alpha_{2}\right), \cdots,\left(s_{n}, \alpha_{n}\right)\right) \\
& =\operatorname{TOWG}\left(\left(s_{1}, \alpha_{1}\right),\left(s_{2}, \alpha_{2}\right), \cdots,\left(s_{n}, \alpha_{n}\right)\right) .
\end{aligned}
$$

Proposition 3. If $\left(s_{j}, \alpha_{j}\right)=(s, \alpha)$ for all $j=1,2, \cdots, n$, then THOWG $\left(\left(s_{1}, \alpha_{1}\right),\left(s_{2}, \alpha_{2}\right), \cdots,\left(s_{n}, \alpha_{n}\right)\right)=(s, \alpha)$.

Proof. It is easy to find the conclusion according to Eq. (9).
Proposition 4. Let $\left(s_{1}, \alpha_{1}\right),\left(s_{2}, \alpha_{2}\right), \cdots,\left(s_{n}, \alpha_{n}\right) \quad$ and $\left(s_{1}^{\prime}, \alpha_{1}^{\prime}\right),\left(s_{2}^{\prime}, \alpha_{2}^{\prime}\right), \cdots,\left(s_{n}^{\prime}, \alpha_{n}^{\prime}\right)$ be two sets of 2-tuples, if $\left(s_{j}, \alpha_{j}\right) \leq\left(s_{j}^{\prime}, \alpha_{j}^{\prime}\right)$ for all $j=1,2, \cdots, n$ and the weight conditions are fixed, then

$$
\begin{aligned}
& \operatorname{THOWG}\left(\left(s_{1}, \alpha_{1}\right),\left(s_{2}, \alpha_{2}\right), \cdots,\left(s_{n}, \alpha_{n}\right)\right) \\
& \leq \operatorname{THOWG}\left(\left(s_{1}^{\prime}, \alpha_{1}^{\prime}\right),\left(s_{2}^{\prime}, \alpha_{2}^{\prime}\right), \cdots,\left(s_{n}^{\prime}, \alpha_{n}^{\prime}\right)\right)
\end{aligned}
$$

Proof. According to Eq. (9), we have

$$
\begin{gathered}
\operatorname{THOWG}\left(\left(s_{1}, \alpha_{1}\right),\left(s_{2}, \alpha_{2}\right), \cdots,\left(s_{n}, \alpha_{n}\right)\right)=\Delta\left(\prod_{j=1}^{n}\left(\Delta^{-1}\left(\widehat{s}_{j}, \widehat{\alpha}_{j}\right)\right)^{\omega_{j}}\right) \\
\operatorname{THOWG}\left(\left(s_{1}^{\prime}, \alpha_{1}^{\prime}\right),\left(s_{2}^{\prime}, \alpha_{2}^{\prime}\right), \cdots,\left(s_{n}^{\prime}, \alpha_{n}^{\prime}\right)\right)=\Delta\left(\prod_{j=1}^{n}\left(\Delta^{-1}\left(\widehat{s}_{j}^{\prime}, \hat{\alpha}_{j}^{\prime}\right)\right)^{\omega_{j}}\right) . \\
\text { If }\left(s_{j}, \alpha_{j}\right) \leq\left(s_{j}^{\prime}, \alpha_{j}^{\prime}\right), \text { then } \\
\Delta^{-1}\left(s_{j}, \alpha_{j}\right) / n w_{j} \leq \Delta^{-1}\left(s_{j}^{\prime}, \alpha_{j}^{\prime}\right) / n w_{j} .
\end{gathered}
$$

So,

$$
\left(\widehat{s}_{j}, \widehat{\alpha}_{j}\right)=\Delta\left(\Delta^{-1}\left(s_{j}, \alpha_{j}\right) / n w_{j}\right) \leq \Delta\left(\Delta^{-1}\left(s_{j}^{\prime}, \alpha_{j}^{\prime}\right) / n w_{j}\right)=\left(\widehat{s}_{j}^{\prime}, \widehat{\alpha}_{j}^{\prime}\right) .
$$

Therefore,

$$
\Delta^{-1}\left(\widehat{s}_{j}, \widehat{\alpha}_{j}\right) \leq \Delta^{-1}\left(\hat{s}_{j}^{\prime}, \hat{\alpha}_{j}^{\prime}\right),
$$

and then,

$$
\Delta\left(\prod_{j=1}^{n}\left(\Delta^{-1}\left(\widehat{s}_{j}, \hat{\alpha}_{j}\right)\right)^{\omega_{j}}\right) \leq \Delta\left(\prod_{j=1}^{n}\left(\Delta^{-1}\left(\widehat{s}_{j}^{\prime}, \hat{\alpha}_{j}^{\prime}\right)\right)^{\omega_{j}}\right) .
$$

Consequently, we derive

$$
\begin{aligned}
& \operatorname{THOWG}\left(\left(s_{1}, \alpha_{1}\right),\left(s_{2}, \alpha_{2}\right), \cdots,\left(s_{n}, \alpha_{n}\right)\right) \\
& \leq \operatorname{THOWG}\left(\left(s_{1}^{\prime}, \alpha_{1}^{\prime}\right),\left(s_{2}^{\prime}, \alpha_{2}^{\prime}\right), \cdots,\left(s_{n}^{\prime}, \alpha_{n}^{\prime}\right)\right) .
\end{aligned}
$$

2) Generating cloud method.

Assume that decision makers' linguistic evaluation scale is $\mathrm{n}$ and $U=\left[X_{\min }, X_{\max }\right]$ is effective universe given by experts, the intermediate normal cloud can be expressed by $C_{0}\left(E x_{0}, E n_{0}, H e_{0}\right)$, then the respective representation of adjacent normal cloud are $C_{-1}\left(E x_{-1}, E n_{-1}, H e_{-1}\right)$, $C_{+1}\left(E x_{+1}, E n_{+1}, H e_{+1}\right), \cdots, \quad C_{-\frac{n-1}{2}}\left(E x_{-\frac{n-1}{2}}, E n_{-\frac{n-1}{2}}, H e_{-\frac{n-1}{2}}\right)$, $C_{+\frac{n-1}{2}}\left(E x_{+\frac{n-1}{2}}, E n_{+\frac{n-1}{2}}, H e_{+\frac{n-1}{2}}\right)$.

Based on golden section technique[23], we present a method that a 2-tuple linguistic can be converted into a corresponding normal cloud. For example, to generate five normal clouds, writing $\xi=\Delta^{-1}\left(s_{j}, \alpha_{j}\right) / j, \quad \eta=\frac{1-\xi}{1+\xi}$, 
$E n^{\prime}=\frac{0.382\left(X_{\max }-X_{\min }\right)}{6}$, then the three number features of normal cloud can be described as follows:

$E x_{0}=\frac{X_{\text {min }}+X_{\text {max }}}{2} \cdot \xi, \quad E x_{-2}=X_{\text {min }} \cdot \xi, \quad E x_{+2}=X_{\text {max }} \cdot \xi$,

$E x_{-1}=\frac{X_{\min }+X_{\max }}{2} \cdot \xi-0.382 \cdot \frac{X_{\min }+X_{\max }}{2} \cdot \xi$

$E x_{+1}=E x_{0}+0.382 \cdot \frac{X_{\min }+X_{\max }}{(\mathrm{n}-3) / 2} \cdot \xi, E n_{0}=0.618(1+\eta) \cdot E n^{\prime}$,

$E n_{-1}=E n_{+1}=0.382(1+\eta) \cdot\left(X_{\text {max }}-X_{\text {min }}\right) / 6$

$E n_{-2}=E n_{+2}=(1+\eta) \cdot E n^{\prime} / 0.618$.

Suppose $H e_{0}$ is known, then $H e_{-1}=H e_{+1}=H e_{0} / 0.618$, $H e_{-2}=H e_{+2}=H e_{+1} / 0.618$.

If $\alpha_{j}=0$, then 2-tuple linguistic degenerates into natural language. In this case, the natural language is converted into a normal cloud via the generating cloud method.

\section{B. The cloud distance and cloud possibility degree}

For the sake of comprehensively considering three number features of normal cloud model, the cloud distance and cloud possibility degree are defined based on "3En rules" of cloud model.

Definition 8[22] Wang\&Zhang Given $Y_{1}, Y_{2}$ over a set $\mathrm{F}$ of one-dimensional normal cloud, a function $d: F \times F \rightarrow R$ with the following properties:

(1) $d\left(Y_{1}, Y_{2}\right) \geq 0, d\left(Y_{2}, Y_{1}\right) \geq 0$,

(2) $d\left(Y_{1}, Y_{2}\right)=d\left(Y_{2}, Y_{1}\right)$,

( 3 ) If for any $Y_{3} \in F, d\left(Y_{1}, Y_{3}\right) \leq d\left(Y_{1}, Y_{2}\right)+d\left(Y_{2}, Y_{3}\right)$, then $d\left(Y_{1}, Y_{2}\right)$ is called the distance of normal cloud.

Let $C_{1}\left(E x_{1}, E n_{1}, H e_{1}\right)$ and $C_{2}\left(E x_{2}, E n_{2}, H e_{2}\right)$ be onedimensional normal cloud in universe $U$, according to " $3 E n$ rules" of normal cloud model, we have the following definition.

Definition 9. The distance $d\left(C_{1}, C_{2}\right)$ of normal cloud between $C_{1}$ and $C_{2}$ is defined as:

$$
d\left(C_{1}, C_{2}\right)=\frac{\underline{d}\left(C_{1}, C_{2}\right)+\bar{d}\left(C_{1}, C_{2}\right)}{2}
$$

where

$$
\underline{d}\left(C_{1}, C_{2}\right)=\left|\left(1-\frac{3 \sqrt{E n_{1}^{2}+H e_{1}^{2}}}{E x_{1}}\right) E x_{1}-\left(1-\frac{3 \sqrt{E n_{2}^{2}+H e_{2}^{2}}}{E x_{2}}\right) E x_{2}\right|
$$

$$
\bar{d}\left(C_{1}, C_{2}\right)=\left|\left(1+\frac{3 \sqrt{E n_{1}^{2}+H e_{1}^{2}}}{E x_{1}}\right) E x_{1}-\left(1+\frac{3 \sqrt{E n_{2}^{2}+H e_{2}^{2}}}{E x_{2}}\right) E x_{2}\right|
$$

Proposition 5. The distance of normal cloud (i.e., Eq.(10))satisfies the following properties:

(1) $d\left(C_{1}, C_{2}\right) \geq 0, d\left(C_{2}, C_{1}\right) \geq 0$,

(2) $d\left(C_{1}, C_{2}\right)=d\left(C_{2}, C_{1}\right)$,

(3) For any $C_{3} \in F, d\left(C_{1}, C_{3}\right) \leq d\left(C_{1}, C_{2}\right)+d\left(C_{2}, C_{3}\right)$.

Proof. (1) (2) It is easy to see that $d\left(C_{1}, C_{2}\right)=d\left(C_{1}, C_{2}\right) \geq 0$

(3) According to definition 9, we have

$$
\begin{aligned}
& \underline{d}\left(C_{1}, C_{3}\right)=\left|\left(1-\frac{3 \sqrt{E n_{1}^{2}+H e_{1}^{2}}}{E x_{1}}\right) E x_{1}-\left(1-\frac{3 \sqrt{E n_{3}^{2}+H e_{3}^{2}}}{E x_{3}}\right) E x_{3}\right| \\
& =\left|\begin{array}{c}
\left(1-\frac{3 \sqrt{E n_{1}^{2}+H e_{1}^{2}}}{E x_{1}}\right) E x_{1}-\left(1-\frac{3 \sqrt{E n_{2}^{2}+H e_{2}^{2}}}{E x_{2}}\right) E x_{2}+ \\
\left(1-\frac{3 \sqrt{E n_{2}^{2}+H e_{2}^{2}}}{E x_{2}}\right) E x_{2}-\left(1-\frac{3 \sqrt{E n_{3}^{2}+H e_{3}^{2}}}{E x_{3}}\right) E x_{3}
\end{array}\right| \\
& \leq\left|\left(1-\frac{3 \sqrt{E n_{1}^{2}+H e_{1}^{2}}}{E x_{1}}\right) E x_{1}-\left(1-\frac{3 \sqrt{E n_{2}^{2}+H e_{2}^{2}}}{E x_{2}}\right) E x_{2}\right|+ \\
& \left|\left(1-\frac{3 \sqrt{E n_{2}^{2}+H e_{2}^{2}}}{E x_{2}}\right) E x_{2}-\left(1-\frac{3 \sqrt{E n_{3}^{2}+H e_{3}^{2}}}{E x_{3}}\right) E x_{3}\right| \\
& =\underline{d}\left(C_{1}, C_{2}\right)+\underline{d}\left(C_{2}, C_{3}\right) .
\end{aligned}
$$

Similarly, we can obtain

$$
\bar{d}\left(C_{1}, C_{3}\right) \leq \bar{d}\left(C_{1}, C_{2}\right)+\bar{d}\left(C_{2}, C_{3}\right) .
$$

Therefore,

$$
\begin{aligned}
& d\left(C_{1}, C_{3}\right)=\frac{1}{2}\left\{\underline{d}\left(C_{1}, C_{3}\right)+\bar{d}\left(C_{1}, C_{3}\right)\right\} \\
& \leq \frac{1}{2}\left\{\underline{d}\left(C_{1}, C_{2}\right)+\underline{d}\left(C_{2}, C_{3}\right)+\bar{d}\left(C_{1}, C_{2}\right)+\bar{d}\left(C_{2}, C_{3}\right)\right\} \\
& =\frac{1}{2}\left\{\underline{d}\left(C_{1}, C_{2}\right)+\bar{d}\left(C_{2}, C_{3}\right)\right\}+\frac{1}{2}\left\{\underline{d}\left(C_{1}, C_{2}\right)+\bar{d}\left(C_{2}, C_{3}\right)\right\} \\
& =d\left(C_{1}, C_{2}\right)+d\left(C_{2}, C_{3}\right)
\end{aligned}
$$

Remark 1.If $E n_{1}=H e_{1}=E n_{2}=H e_{2}=0$, then normal cloud will degenerate into real number, in this case, $d\left(C_{1}, C_{2}\right)=\left|E x_{1}-E x_{2}\right|$. 
Suppose that $\mathrm{C}_{1}\left(E x_{1}, E n_{1}, H e_{1}\right)$ and $\mathrm{C}_{2}\left(E x_{2}, E n_{2}, H e_{2}\right)$ are two one-dimensional normal clouds in universe $\mathrm{U}$, positive ideal cloud $C^{*}\left(\max _{i} E x_{i}, \min _{i} E n_{i}, \min _{i} H e_{i}\right), i=1,2$.

Definition 10. The cloud possibility degree is defined as

$$
p\left(C_{1} \geq C_{2}\right)=1-\frac{d\left(C^{*}, C_{1}\right)}{d\left(C^{*}, C_{1}\right)+d\left(C^{*}, C_{2}\right)},
$$

where $d\left(C^{*}, C_{1}\right)$ and $d\left(C^{*}, C_{2}\right)$ are the distances between positive ideal cloud $\mathrm{C}^{*}$ and $\mathrm{C}_{1}, \mathrm{C}_{2}$, respectively.

Remark 2. - we find that if $p\left(C_{1} \geq C_{2}\right) \geq 0.5$, then $C_{1} \geq C_{2}$, otherwise $C_{1}<C_{2}$.

- In addition, it is easy to conclude that:

(1) $0 \leq p\left(C_{1} \geq C_{2}\right) \leq 1$;

(2) $p\left(C_{1} \geq C_{2}\right)=1 \Leftrightarrow C^{*}=C_{1}, p\left(C_{1} \geq C_{2}\right)=0 \Leftrightarrow C^{*}=C_{2}$;

(3) $p\left(C_{1} \geq C_{2}\right)+p\left(C_{1}<C_{2}\right)=1$, particularly, $p\left(C_{1} \geq C_{1}\right)=1$;

(4) If $p\left(C_{1} \geq C_{2}\right)=p\left(C_{1} \leq C_{2}\right)=0.5$, then $C_{1}=C_{2}$.

Proposition 6. Suppose that $\mathrm{C}_{1}\left(E x_{1}, E n_{1}, H e_{1}\right)$ and $\mathrm{C}_{2}\left(E x_{2}, E n_{2}, H e_{2}\right)$ are two one-dimensional normal clouds in universe $\mathrm{U}$, if $E x_{1}=E x_{2}, E n_{1} \leq E n_{2}, H e_{1} \leq H e_{2}$, then $C_{1} \geq C_{2}$.

Proof. If $E x_{1}=E x_{2}, E n_{1} \leq E n_{2}, H e_{1} \leq H e_{2}$, then the positive ideal cloud is $C^{*}\left(E x_{1}, E n_{1}, H e_{1}\right)$.

According to definitions 9 , we have $d\left(C^{*}, C_{1}\right)=0$. that

Consequently, by applying Eq. (11), it can be concluded

$$
p\left(C_{1} \geq C_{2}\right)=1-\frac{d\left(C^{*}, C_{1}\right)}{d\left(C^{*}, C_{1}\right)+d\left(C^{*}, C_{2}\right)}=1 .
$$

Thus, $C_{1} \geq C_{2}$.

\section{The cloud prospect value function}

According to Eqs.(5), (10) and (11), we can define the cloud prospect value function.

Definition 11. Suppose that $C_{i}\left(E x_{i}, E n_{i}, H e_{i}\right)$ and $C_{0}\left(E x_{0}, E n_{0}, H e_{0}\right)$ are two one-dimensional normal clouds in universe $U$, the prospect value function of cloud $C_{i}$ is defined as:

$$
V\left(C_{i}\right)==\left\{\begin{array}{l}
\left(d\left(C_{i}, C_{0}\right)\right)^{\alpha} \quad, C_{i} \geq C_{0} \\
-\lambda\left(d\left(C_{i}, C_{0}\right)\right)^{\beta}, C_{i}<C_{0}
\end{array},\right.
$$

where cloud $\mathrm{C}_{0}$ is reference point.
Remark 3. Here $C_{i} \geq C_{0}$ (or $C_{i}<C_{0}$ ) can be determined via cloud possibility degree (11).

\section{AN APPROACH OF 2-TUPLE LINGUISTIC FUZZY STOCHASTIC MULTI-ATTRIBUTES GROUP DECISION-MAKING}

This section presents an approach to dealing with the 2tuple linguistic fuzzy stochastic MAGDM problems where the attributes weights are incompletely known.

\section{A. Problem description}

Let $Z=\left\{Z_{1}, \cdots, Z_{i}, \cdots, Z_{m}\right\}$ be a discrete set of alternatives, $S=\left\{S_{1}, \cdots, S_{j}, \cdots, S_{n}\right\} \quad$ a finite set of attributes, and $E=\left\{E_{1}, \cdots, E_{l}, \cdots E_{t}\right\}$ is a finite set of decision makers. Assume that the weight vector of attributes is $w=\left(w_{1}, \cdots, w_{j}, \cdots w_{n}\right)^{T}$ such that $\sum_{j=1}^{n} w_{j}=1, w_{j} \geq 0, \quad$ and $\lambda=\left(\lambda_{1}, \cdots, \lambda_{l}, \cdots, \lambda_{t}\right)^{T}$ is the weight vector of decision makers with $\sum_{l=1}^{t} \lambda_{l}=1, \lambda_{l} \geq 0$. Denoted by $\theta=\left(\theta_{1}, \cdots, \theta_{k}, \cdots, \theta_{s}\right) \quad$ the possible status, and $p_{k}$ the probability of the status $\theta_{k}$, where $0 \leq p_{k} \leq 1, \sum_{k=1}^{s} p_{k}=1$.

The decision makers $E_{l}(l=1,2, \cdots, t)$ provide their preference values for each alternative on each criterion under status $\theta_{k}(k=1,2, \cdots s)$, and consequently construct the decision matrix $R_{l}=\left(r_{i j k}^{(\mathrm{I})}\right)_{m \times n}$, where $r_{i j k}^{(\mathrm{I})}$ takes the form of linguistic variable.

In addition, $w=\left(w_{1}, \cdots, w_{j}, \cdots w_{n}\right)^{T} \in H$ be the weight vector of attributes, where $H$ be the set of the incompletely known attributes weights. In general, $H$ can be expressed as one of the combinations of the following form: $a_{i} \leq w_{i}, w_{i} \geq b_{i}, \gamma_{i} \leq w_{i} \leq \gamma_{i}+\varepsilon_{i}, w_{i} \geq \beta w_{j}, w_{i}-w_{j} \geq \alpha$, $\theta_{i} w_{j} \leq\left(\theta_{i}+\varepsilon_{i}\right) w_{i}$ and $w_{i}-w_{j} \geq w_{k}-w_{l}(j \neq k \neq l)$.

\section{B. The decision-making approach}

In this section, we propose a new approach to solve the fuzzy stochastic MAGDM problems with 2-tuple linguistic assessments. An algorithm and process of the MAGDM problems with 2-tuple linguistic assessments may be given as follows.

Step 1.Derive 2-tuple linguistic decision matrix.

Transform linguistic decision matrix $R_{l}=\left(r_{i j k}{ }^{(l)}\right)_{m \times n}$ into 2tuple linguistic decision matrix $\tilde{R}_{l}=\left(r_{i j k}{ }^{(l)}, 0\right)_{m \times n}$ by applying Eq. (3).

Step 2.Aggregate decision matrices. 
Utilize the decision information given in matrix $\tilde{R}_{l}$, and THOWG operator which has associated weighting vector $\omega=\left(\omega_{1}, \cdots, \omega_{l}, \cdots \omega_{t}\right)^{T}$

$$
\begin{aligned}
x_{i j k}=\left(r_{i j k}, \alpha_{i j k}\right)= & \operatorname{THOWG}\left(\left(r_{i j k}^{(1)}, 0\right), \cdots\left(r_{i j k}^{(l)}, 0\right), \cdots,\left(r_{i j k}^{(t)}, 0\right)\right) \\
& =\Delta\left(\prod_{l=1}^{t}\left(\Delta^{-1}\left(\hat{r}_{i j k}^{(l)}, \hat{\alpha}_{i j k}^{(l)}\right)\right)^{\omega_{l}}\right), \\
& r_{i j k}^{(l)} \in S, \alpha_{i j k}^{(l)} \in[-0.5 .0 .5), k=1,2, \cdots \mathrm{s} .
\end{aligned}
$$

to aggregate all the decision matrices $\tilde{R}_{l}(l=1,2, \cdots, t)$ into a collective decision matrix $\tilde{R}=\left(r_{i j k}, \alpha_{i j k}\right)_{m \times n}$, where $\left(\hat{r}_{i j k}^{(l)}, \hat{\alpha}_{i j k}^{(l)}\right)$ is jth element of $\left\{\Delta\left(\Delta^{-1}\left(r_{i j k}^{(1)}, 0\right) / t \lambda_{1}\right), \Delta\left(\Delta^{-1}\left(r_{i j k}^{(2)}, 0\right) / t \lambda_{2}\right), \cdots\right.$, $\left.\Delta\left(\Delta^{-1}\left(r_{i j k}^{(t)}, 0\right) / t \lambda_{t}\right)\right\}$ according to descending order, and $\lambda=\left(\lambda_{1}, \lambda_{2}, \cdots, \lambda_{t}\right)^{T}$ the weighting vector of decision makers.

Step 3.Calculate cloud decision matrix.

According to generating cloud method, the collective decision matrix $\tilde{R}$ is converted into the corresponding normal cloud decision matrix $R$.

Step 4.Compute the cloud prospect function value.

Based on Eq. (4), it can be calculated the prospect function value $v_{i j}$ of the jth criterion under the ith alternative by selecting all other alternatives as the reference point. Therefore, we obtain the prospect function value of cloud model shown as follows:

$$
V_{i j}(C)=\sum_{k=1}^{s} \sum_{u=1, u \neq i}^{m} v\left(C_{u j i}^{k}\right) \pi_{u j i}\left(p_{k}\right),
$$

where,

$$
\begin{gathered}
v\left(C_{u j i}^{k}\right)=\left\{\begin{array}{ll}
\left(d\left(C_{u j}^{k}, C_{i j}^{k}\right)\right)^{\alpha} & , C_{i j}^{k} \geq C_{u j}^{k} \\
-\lambda\left(d\left(C_{u j}^{k}, C_{i j}^{k}\right)\right)^{\beta} & , C_{i j}^{k}<C_{u j}^{k}
\end{array} ;\right. \\
\pi_{l j i}\left(p_{k}\right)= \begin{cases}\frac{p_{k}^{\gamma}}{\left(p_{k}^{\gamma}+\left(1-p_{k}\right)^{\gamma}\right)^{\frac{1}{\gamma}}}, & C_{i j}^{k} \geq C_{u j}^{k} \\
\frac{p_{k}^{\delta}}{\left(p_{k}^{\delta}+\left(1-p_{k}\right)^{\delta}\right)^{\frac{1}{\delta}}} & , C_{i j}^{k}<C_{u j}^{k}\end{cases}
\end{gathered}
$$

Step 5.Determine the attributes weights.

Considering that the principle of maximizing the integrated cloud prospect value is consistent with the habit of decision maker, in other words, decision maker always maximizes his/her benefits (or minimizes his/her costs), we can construct the following optimization model to determine the attributes weights by applying the method of maximizing the integrated cloud prospect value.

$$
\begin{array}{ll}
\max & M_{2}=V \cdot w \\
\text { s.t. } & \sum_{j=1}^{n} w_{j}=1, \\
& w_{j} \in H, \\
& w_{j}>0,(j=1,2, \cdots, n),
\end{array}
$$

where $\mathrm{V}$ is the integrated cloud prospect value, $w=\left(w_{1}, w_{2}, \cdots, w_{n}\right)^{T}$ an attributes weight vector, and $H$ the set of the attributes weights which are incompletely known.

It can be utilized mathematic software (e.g., Matlab or Lingo) to solve the above optimization model and get the desirable attributes weight vector $w=\left(w_{1}, w_{2}, \ldots, w_{n}\right)^{T}$.

Step 6.Derive the integrated cloud prospect value.

By applying Eqs. (14) and (15), we can derive the integrated cloud prospect value shown as follows:

$$
V_{i}=\sum_{j=1}^{n} v_{i j} w_{j}(i=1, \cdots, m),
$$

where $V_{i}$ is the integrated cloud prospect value.

Step 7.Rank the order of alternatives.

According to the integrated cloud prospect value $V_{i}(i=1, \cdots, m)$, one can rank the order of alternatives with the fact that the larger the value of $V_{i}(i=1, \cdots, m)$ is, the better the alternative is.

\section{NUMERICAL EXAMPLE}

The future development trend of electric power grid is smart grid, which possesses such features as being clean, secure, economic, and so on. It is necessary to carry out risk management in the construction of smart grids in China due to the more complicated operation environment. Let us suppose a grid company needs to manage, there is a panel with four possible alternatives to elect: $Z_{1}, Z_{2}, Z_{3}, Z_{4}$. The grid company must include the following three attributes while making the choice:(1) $G_{1}$ expresses the police risk, (2) $G_{2}$ stands for the technology risk, (3) $G_{3}$ denotes the market risk. According to the market forecast, there are three natural statuses for the attributes: good $\left(\theta_{1}\right)$, medium $\left(\theta_{2}\right)$ and poor $\left(\theta_{3}\right)$, and the corresponding probability of each status are $0.3,0.5$ and 0.2 , respectively. In addition, the experts' evaluation can provide information on the weight set which is shown as follows: $H=\left\{0.2 \leq w_{1} \leq 0.5,0.1 \leq w_{2} \leq 0.4,0.4 \leq w_{3} \leq 0.7\right\}$. The four possible alternatives are to be evaluated using the linguistic term set $\mathrm{S}=\left\{\mathrm{s}_{0}=\right.$ extremely poor, $\mathrm{s}_{1}=$ poor, $\mathrm{s}_{2}=$ fair, $\mathrm{s}_{3}=$ good, $\mathrm{s}_{4}=$ extremely good $\}$ by the three decision makers under the above three attributes and three natural statuses. The assessments for alternatives arising from questionnaire investigation to the experts are shown in Table I. 
TABLE I. LINGUISTIC DECISION MATRIX $R_{l}$

\begin{tabular}{|c|c|c|c|c|c|c|}
\hline & & & $Z_{1}$ & $\mathrm{Z}_{2}$ & $Z_{3}$ & $Z_{4}$ \\
\hline \multirow{9}{*}{$E_{1}$} & \multirow{3}{*}{$\theta_{1}=0.3$} & $\mathrm{G}_{1}$ & EG & $\mathrm{F}$ & $F$ & $\mathrm{G}$ \\
\hline & & $\mathrm{G}_{2}$ & $\mathrm{G}$ & EG & G & EG \\
\hline & & $\mathrm{G}_{3}$ & EG & G & EG & EG \\
\hline & \multirow{4}{*}{$\theta_{2}=0.5$} & $\mathrm{G}_{1}$ & $\mathrm{~F}$ & $\mathrm{P}$ & $\mathrm{P}$ & $\mathrm{F}$ \\
\hline & & $\mathrm{G}_{2}$ & $\mathrm{~F}$ & $\mathrm{P}$ & $\mathrm{F}$ & $P$ \\
\hline & & $\mathrm{G}_{3}$ & G & $\mathrm{F}$ & EG & G \\
\hline & & $\mathrm{G}_{1}$ & EG & $\mathrm{F}$ & $\mathrm{P}$ & $\mathrm{G}$ \\
\hline & \multirow{2}{*}{$\theta_{3}=0.2$} & $\mathrm{G}_{2}$ & EP & $\mathrm{F}$ & $\mathrm{F}$ & EP \\
\hline & & $\mathrm{G}_{3}$ & $\mathrm{~F}$ & $\mathrm{~F}$ & $\mathrm{~F}$ & EG \\
\hline \multirow{9}{*}{$\mathrm{E}_{2}$} & \multirow{3}{*}{$\theta_{1}=0.3$} & $\mathrm{G}_{1}$ & G & $\mathrm{P}$ & G & $\mathrm{G}$ \\
\hline & & $\mathrm{G}_{2}$ & G & G & $\mathrm{F}$ & $\mathrm{G}$ \\
\hline & & $\mathrm{G}_{3}$ & EG & EG & G & EG \\
\hline & \multirow{3}{*}{$\theta_{2}=0.5$} & $\mathrm{G}_{1}$ & F & $P$ & $\mathrm{~F}$ & $P$ \\
\hline & & $\mathrm{G}_{2}$ & $\mathrm{P}$ & $\mathrm{P}$ & $\mathrm{F}$ & $\mathrm{F}$ \\
\hline & & $\mathrm{G}_{3}$ & G & $\mathrm{F}$ & $\mathrm{G}$ & $\mathrm{G}$ \\
\hline & & $\mathrm{G}_{1}$ & EG & $\mathrm{F}$ & $\mathrm{P}$ & $\mathrm{G}$ \\
\hline & \multirow{2}{*}{$\theta_{3}=0.2$} & $\mathrm{G}_{2}$ & $\mathrm{P}$ & $\mathrm{F}$ & G & $\mathrm{P}$ \\
\hline & & $\mathrm{G}_{3}$ & $\mathrm{~F}$ & $\mathrm{P}$ & $\mathrm{F}$ & $\mathrm{G}$ \\
\hline \multirow{9}{*}{$E_{3}$} & \multirow{3}{*}{$\theta_{1}=0.3$} & $\mathrm{G}_{1}$ & EG & $\mathrm{F}$ & $\mathrm{F}$ & $\mathrm{G}$ \\
\hline & & $\mathrm{G}_{2}$ & $\mathrm{G}$ & EG & $\mathrm{G}$ & EG \\
\hline & & $\mathrm{G}_{3}$ & EG & G & EG & EG \\
\hline & \multirow{3}{*}{$\theta_{2}=0.5$} & $\mathrm{G}_{1}$ & F & $P$ & $\mathrm{P}$ & $\mathrm{F}$ \\
\hline & & $\mathrm{G}_{2}$ & $\mathrm{~F}$ & $\mathrm{P}$ & F & $\mathrm{P}$ \\
\hline & & $\mathrm{G}_{3}$ & G & $\mathrm{F}$ & EG & $\mathrm{G}$ \\
\hline & \multirow{3}{*}{$\theta_{3}=0.2$} & $\mathrm{G}_{1}$ & EG & $\mathrm{F}$ & G & $\mathrm{G}$ \\
\hline & & $\mathrm{G}_{2}$ & EP & $\mathrm{F}$ & $\mathrm{F}$ & EP \\
\hline & & $\mathrm{G}_{3}$ & $\mathrm{~F}$ & $\mathrm{~F}$ & $\mathrm{~F}$ & EG \\
\hline
\end{tabular}

(1) Derive 2-tuple linguistic decision matrix.

Transform the linguistic decision matrix $R_{l}=\left(r_{i j k}{ }^{(\mathrm{l})}\right)_{4 \times 3}, l=1,2,3 ; k=1,2,3$ given in Table 1 into 2-tuple linguistic decision matrix $\tilde{R}_{l}=\left(r_{i j k}{ }^{(1)}, 0\right)_{4 \times 3}, l=1,2,3 ; k=1,2,3$ given in Table II.

\section{(2) Aggregate decision matrices.}

Based on THOWG operator, calculating the collective 2tuple linguistic decision matrix $\tilde{R}=\left(r_{i j k}, \alpha_{i j k}\right)_{4 \times 3}, k=1,2,3$ given in Table III, where $\omega=(0.3,0.3,0.4)^{T}$ is the associated weighting vector with THOWG operator and $\lambda=(0.3,0.4,0.3)^{T}$ is the weighting vector of decision makers.

(3)Calculate cloud decision matrix.

According to generating cloud method, the collective 2tuple linguistic decision matrix is converted into the corresponding normal cloud decision matrix $R$ given in Table IV.

(4)Compute the cloud prospect function value.

By using Eq.(14), where $\alpha=\beta=0.88, \lambda=2.25, \gamma=0.61$ and $\delta=0.69$, we get the cloud prospect matrix shown as follows:

$$
V=\left(\begin{array}{ccc}
57.733 & -51.692 & -11.627 \\
-92.011 & -6.619 & -119.263 \\
-70.486 & -4.238 & 1.868 \\
-8.382 & -26.565 & 21.594
\end{array}\right)
$$

TABLE II. 2-TUPLE LINGUISTIC DECISION MATRIX $\tilde{R}_{l}$

\begin{tabular}{|c|c|c|c|c|c|c|}
\hline & & & $Z_{1}$ & $Z_{2}$ & $Z_{3}$ & $Z_{4}$ \\
\hline \multirow{9}{*}{$E_{1}$} & \multirow{3}{*}{$\theta_{1}=0.3$} & $\mathrm{G}_{1}$ & $(\mathrm{EG}, 0)$ & $(\mathrm{F}, 0)$ & $(\mathrm{F}, 0)$ & $(\mathrm{G}, 0)$ \\
\hline & & $\mathrm{G}_{2}$ & $(\mathrm{G}, 0)$ & $(\mathrm{EG}, 0)$ & $(\mathrm{G}, 0)$ & $(\mathrm{EG}, 0)$ \\
\hline & & $\mathrm{G}_{3}$ & $(\mathrm{EG}, 0)$ & $(\mathrm{G}, 0)$ & $(\mathrm{EG}, 0)$ & $(\mathrm{EG}, 0)$ \\
\hline & \multirow{3}{*}{$\theta_{2}=0.5$} & $\mathrm{G}_{1}$ & $(\mathrm{~F}, 0)$ & $(\mathrm{P}, 0)$ & $(\mathrm{P}, 0)$ & $(\mathrm{F}, 0)$ \\
\hline & & $\mathrm{G}_{2}$ & $(\mathrm{~F}, 0)$ & $(\mathrm{P}, 0)$ & $(\mathrm{F}, 0)$ & $(\mathrm{P}, 0)$ \\
\hline & & $\mathrm{G}_{3}$ & $(\mathrm{G}, 0)$ & $(\mathrm{F}, 0)$ & $(\mathrm{EG}, 0)$ & $(\mathrm{G}, 0)$ \\
\hline & \multirow{3}{*}{$\theta_{3}=0.2$} & $\mathrm{G}_{1}$ & $(\mathrm{EG}, 0)$ & $(\mathrm{F}, 0)$ & $(\mathrm{P}, 0)$ & $(\mathrm{G}, 0)$ \\
\hline & & $\mathrm{G}_{2}$ & $(\mathrm{EP}, 0)$ & $(\mathrm{F}, 0)$ & $(\mathrm{F}, 0)$ & $(\mathrm{EP}, 0)$ \\
\hline & & $\mathrm{G}_{3}$ & $(\mathrm{~F}, 0)$ & $(\mathrm{F}, 0)$ & $(\mathrm{F}, 0)$ & $(\mathrm{EG}, 0)$ \\
\hline \multirow{9}{*}{$\mathrm{E}_{2}$} & \multirow{3}{*}{$\theta_{1}=0.3$} & $\mathrm{G}_{1}$ & $(\mathrm{G}, 0)$ & $(\mathrm{P}, 0)$ & $(G, 0)$ & $(\mathrm{G}, 0)$ \\
\hline & & $\mathrm{G}_{2}$ & $(\mathrm{G}, 0)$ & $(\mathrm{G}, 0)$ & $(\mathrm{F}, 0)$ & $(\mathrm{G}, 0)$ \\
\hline & & $\mathrm{G}_{3}$ & $(\mathrm{EG}, 0)$ & $(\mathrm{EG}, 0)$ & $(\mathrm{G}, 0)$ & $(\mathrm{EG}, 0)$ \\
\hline & \multirow{3}{*}{$\theta_{2}=0.5$} & $\mathrm{G}_{1}$ & $(\mathrm{~F}, 0)$ & $(\mathrm{P}, 0)$ & $(\mathrm{F}, 0)$ & $(\mathrm{P}, 0)$ \\
\hline & & $\mathrm{G}_{2}$ & $(\mathrm{P}, 0)$ & $(\mathrm{P}, 0)$ & $(\mathrm{F}, 0)$ & $(\mathrm{F}, 0)$ \\
\hline & & $\mathrm{G}_{3}$ & $(\mathrm{G}, 0)$ & $(\mathrm{F}, 0)$ & $(\mathrm{G}, 0)$ & $(\mathrm{G}, 0)$ \\
\hline & \multirow{3}{*}{$\theta_{3}=0.2$} & $\mathrm{G}_{1}$ & $(\mathrm{EG}, 0)$ & $(\mathrm{F}, 0)$ & $(\mathrm{P}, 0)$ & $(\mathrm{G}, 0)$ \\
\hline & & $\mathrm{G}_{2}$ & $(\mathrm{P}, 0)$ & $(\mathrm{F}, 0)$ & $(\mathrm{G}, 0)$ & $(\mathrm{P}, 0)$ \\
\hline & & $\mathrm{G}_{3}$ & $(\mathrm{~F}, 0)$ & $(\mathrm{P}, 0)$ & $(\mathrm{F}, 0)$ & $(\mathrm{G}, 0)$ \\
\hline \multirow{9}{*}{$\mathrm{E}_{3}$} & \multirow{3}{*}{$\theta_{1}=0.3$} & $\mathrm{G}_{1}$ & $(\mathrm{EG}, 0)$ & $(\mathrm{F}, 0)$ & $(\mathrm{F}, 0)$ & $(\mathrm{G}, 0)$ \\
\hline & & $\mathrm{G}_{2}$ & $(\mathrm{G}, 0)$ & $(\mathrm{EG}, 0)$ & $(\mathrm{G}, 0)$ & $(\mathrm{EG}, 0)$ \\
\hline & & $\mathrm{G}_{3}$ & $(\mathrm{EG}, 0)$ & $(\mathrm{G}, 0)$ & $(\mathrm{EG}, 0)$ & $(\mathrm{EG}, 0)$ \\
\hline & \multirow{3}{*}{$\theta_{2}=0.5$} & $\mathrm{G}_{1}$ & $(\mathrm{~F}, 0)$ & $(\mathrm{P}, 0)$ & $(\mathrm{P}, 0)$ & $(\mathrm{F}, 0)$ \\
\hline & & $\mathrm{G}_{2}$ & $(\mathrm{~F}, 0)$ & $(\mathrm{P}, 0)$ & $(\mathrm{F}, 0)$ & $(\mathrm{P}, 0)$ \\
\hline & & $\mathrm{G}_{3}$ & $(\mathrm{G}, 0)$ & $(\mathrm{F}, 0)$ & $(\mathrm{EG}, 0)$ & $(\mathrm{G}, 0)$ \\
\hline & \multirow{3}{*}{$\theta_{3}=0.2$} & $\mathrm{G}_{1}$ & $(\mathrm{EG}, 0)$ & $(\mathrm{F}, 0)$ & $(\mathrm{G}, 0)$ & $(\mathrm{G}, 0)$ \\
\hline & & $\mathrm{G}_{2}$ & $(\mathrm{EP}, 0)$ & $(\mathrm{F}, 0)$ & $(\mathrm{F}, 0)$ & $(\mathrm{EP}, 0)$ \\
\hline & & $\mathrm{G}_{3}$ & $(\mathrm{~F}, 0)$ & $(\mathrm{F}, 0)$ & $(\mathrm{F}, 0)$ & $(E G, 0)$ \\
\hline
\end{tabular}

TABLE III. COLLECTIVE DECISION MATRIX $\tilde{R}$

\begin{tabular}{ccccccc}
\hline & & & $\mathrm{Z}_{1}$ & $\mathrm{Z}_{2}$ & $\mathrm{Z}_{3}$ & $\mathrm{Z}_{4}$ \\
\hline & & $\mathrm{G}_{1}$ & $(\mathrm{EG},-0.47)$ & $(\mathrm{F},-0.5)$ & $(\mathrm{F}, 0.3)$ & $(\mathrm{G},-0.03)$ \\
& $\theta_{1}=0.3$ & $\mathrm{G}_{2}$ & $(\mathrm{G},-0.03)$ & $(\mathrm{EG},-0.47)$ & $(\mathrm{G},-0.47)$ & $(\mathrm{EG},-0.47)$ \\
& & $\mathrm{G}_{3}$ & $(\mathrm{EG},-0.04)$ & $(\mathrm{G}, 0.33)$ & $(\mathrm{EG},-0.47)$ & $(\mathrm{EG},-0.04)$ \\
& & $\mathrm{G}_{1}$ & $(\mathrm{~F},-0.02)$ & $(\mathrm{P},-0.01)$ & $(\mathrm{P}, 0.25)$ & $(\mathrm{F},-0.5)$ \\
$\mathrm{E}_{1}$ & $\theta_{2}=0.5$ & $\mathrm{G}_{2}$ & $(\mathrm{~F},-0.5)$ & $(\mathrm{P},-0.01)$ & $(\mathrm{F},-0.02)$ & $(\mathrm{P}, 0.25)$ \\
& & $\mathrm{G}_{3}$ & $(\mathrm{G},-0.03)$ & $(\mathrm{F},-0.02)$ & $(\mathrm{EG},-0.47)$ & $(\mathrm{G},-0.03)$ \\
& & $\mathrm{G}_{1}$ & $(\mathrm{EG}, 0)$ & $(\mathrm{F},-0.02)$ & $(\mathrm{P},-0.01)$ & $(\mathrm{G},-0.03)$ \\
& $\theta_{3}=0.2$ & $\mathrm{G}_{2}$ & $(\mathrm{P},-0.5)$ & $(\mathrm{F},-0.02)$ & $(\mathrm{F}, 0.3)$ & $(\mathrm{EP},-0.5)$ \\
& $\mathrm{G}_{3}$ & $(\mathrm{~F},-0.02)$ & $(\mathrm{F},-0.5)$ & $(\mathrm{F},-0.02)$ & $(\mathrm{EG},-0.47)$ \\
\hline
\end{tabular}

(5)Derive the attributes weights.

Based on the model (15), derive the optimization attributes weights $w=(0.20,0.40,0.40)$ by applying the software of Lingo 11.0.

(6)Rank the order of alternatives.

Ranking all the alternatives $Z_{i}(i=1,2,3,4)$ in accordance with the integrated cloud prospect value: $V_{1}=-13.781, V_{2}=-68.755, V_{3}=-15.045, V_{4}=-3.665, \quad$ and thus the most desirable alternative is $Z_{4}$.

In order to compare the effectiveness of our approach with the traditional 2-tuple linguistic MAGDM approach, we select the approach of Wei[4], which is one of the classical 2-tuple linguistic MAGDM approaches, as a comparison. We take advantage of the same data in the throughout calculation process which are shown in Table III. On the other hand, it is worth noting that the attributes weights of Wei[4] are 
TABLE IV. COLLECTIVE DECISION MATRIX $\tilde{R}$

\begin{tabular}{|c|c|c|c|}
\hline & & $Z_{1}$ & $Z_{2}$ \\
\hline \multirow{3}{*}{$\theta_{1}$} & $G_{1}$ & $C_{+2}(88,10.96,0.26)$ & $C_{0}(37.50,4.49,0.10)$ \\
\hline & $G_{2}$ & $C_{+1}(68.41,6.40,0.16)$ & $C_{+2}(88,10.96,0.26)$ \\
\hline & $G_{3}$ & $C_{+2}(99,10.35,0.26)$ & $C_{+1}(69.79,6.33,0.16)$ \\
\hline \multirow{3}{*}{$\theta_{2}$} & $G_{1}$ & $C_{0}(49.50,3.95,0.10)$ & $C_{-1}(30.59,6.40,0.16)$ \\
\hline & $G_{2}$ & $C_{0}(37.50,4.49,0.10)$ & $C_{-1}(30.59,6.40,0.16)$ \\
\hline & $G_{3}$ & $C_{+1}(68.41,6.40,0.16)$ & $C_{0}(49.50,3.95,0.10)$ \\
\hline \multirow{4}{*}{$\theta_{3}$} & $G_{1}$ & $C_{+2}(99,10.35,0.26)$ & $C_{0}(49.50,3.95,0.10)$ \\
\hline & $G_{2}$ & $C_{-1}(15.45,8.47,0.16)$ & $C_{0}(49.50,3.95,0.10)$ \\
\hline & $G_{3}$ & $C_{0}(49.50,3.95,0.10)$ & $C_{0}(37.50,4.49,0.10)$ \\
\hline & & $Z_{3}$ & $Z_{4}$ \\
\hline \multirow{3}{*}{$\theta_{1}$} & $G_{1}$ & $C_{0}(57.50,3.66,0.10)$ & $C_{+1}(68.41,6.40,0.16)$ \\
\hline & $G_{2}$ & $C_{+1}(58.25,6.91,0.16)$ & $C_{+2}(88,10.96,0.26)$ \\
\hline & $G_{3}$ & $C_{+2}(88,10.96,0.26)$ & $C_{+2}(99,10.35,0.26)$ \\
\hline \multirow{3}{*}{$\theta_{2}$} & $G_{1}$ & $C_{-1}(38.63,5.67,0.16)$ & $C_{0}(37.50,4.49,0.10)$ \\
\hline & $G_{2}$ & $C_{0}(49.50,3.95,0.10)$ & $C_{-1}(38.63,5.67,0.16)$ \\
\hline & $G_{3}$ & $C_{+2}(88,10.96,0.26)$ & $C_{+1}(68.41,6.40,0.16)$ \\
\hline \multirow{3}{*}{$\theta_{3}$} & $G_{1}$ & $C_{-1}(30.59,6.40,0.16)$ & $C_{+1}(68.41,6.40,0.16)$ \\
\hline & $G_{2}$ & $C_{0}(57.50,3.66,0.10)$ & $C_{-1}(15.45,8.47,0.16)$ \\
\hline & $G_{3}$ & $C_{0}(49.50,3.95,0.10)$ & $C_{+2}(88,10.96,0.26)$ \\
\hline
\end{tabular}

obtained by the maximizing deviation method, which does not take into account the risk attitude of decision makers, as a result, we take $w=(0.20,0.40,0.40)^{T}$ which is just our optimization attributes weights determined by step 5 .

Table $\mathrm{V}$ shows the results obtained by the positive ideal relational degree of each alternative of Wei[4] under good status, medium status and poor status, respectively.

We find that from Table $\mathrm{V}$ there are three different results under the given risk statuses. That is, the alternative $Z_{4}$ is the best under the good status or poor status, whereas the best alternative is $Z_{3}$ under the medium risk. Therefore, it can be concluded that the approach of Wei[4] can't derive a uniformed conclusion under different risk statuses. Our method, however, integrates the statuses of risks (good, medium and poor) into a decision-making matrix by using Prospect theory, and consequently can provide an exact conclusion.

\section{CONCLUSIONS}

This paper has investigated the fuzzy stochastic MAGDM problems where the attributes values of the alternatives are 2tuple linguistic and the information of attributes weights is incompletely known. An approach was provided which is based on Prospect theory and Cloud model. A new 2-tuple aggregation operator was developed so as to aggregate the evaluation value into group's comprehensive evaluation information. In addition, considering the fuzziness and randomness of information, a generating cloud method was

TABLE V. THE RESUlt DERIVED BY THE METHOD OF WeI (2010A)

\begin{tabular}{cccc}
\hline & good $\left(\theta_{1}\right)$ & medium $\left(\theta_{2}\right)$ & poor $\left(\theta_{3}\right)$ \\
\hline$C_{1}$ & $(L,-0.144)$ & $(L,-0.320)$ & $(L,-0.424)$ \\
$C_{2}$ & $(L,-0.286)$ & $(N, 0.402)$ & $(L,-0.438)$ \\
$C_{3}$ & $(L,-0.494)$ & $(L,-0.098)$ & $(L,-0.338)$ \\
$C_{4}$ & $(L,-0.072)$ & $(L,-0.440)$ & $(L,-0.300)$ \\
$\begin{array}{c}\text { Sort } \\
\text { order }\end{array}$ & $Z_{4} \succ Z_{1} \succ Z_{2} \succ Z_{3}$ & $Z_{4} \succ Z_{1} \succ Z_{2} \succ Z_{3}$ & $Z_{4} \succ Z_{1} \succ Z_{2} \succ Z_{3}$ \\
\hline
\end{tabular}

proposed that a 2-tuple linguistic is converted into a corresponding normal cloud. Furthermore, based on Prospect theory, THOWG operator and Cloud model, a new approach was presented to solve the 2-tuple linguistic fuzzy stochastic MAGDM problems. Finally, the feasibility and validity of this approach were illustrated by an example.

In the further research about the 2-tuple linguistic fuzzy stochastic MAGDM problems, it would be very interesting to extend our analysis to the case of more sophisticated situation, such as the dynamic group decision, etc. Nevertheless, we leave that point to future research, since our methodology cannot be applied to that extended framework, which will result in more sophisticated calculation and which we cannot tackle it here.

\section{REFERENCES}

[1] Herrera F, Martinez L, A 2-tuple fuzzy linguistic representation model for computing with words. IEEE Transactions on Fuzzy Systems 2000,8,pp:746-752.

[2] Herrera F, Martinez L, A model based on linguistic 2-tuple for dealing with multi-granular hierarchical linguistic contexts in multi-expert decision-making. IEEE Transactions on Systems, Man, and Cybernetics, 2001,31,pp:227-234.

[3] Wei GW, A method for multiple attribute group decision making based on the ET-WG and ET-OWG operators with 2-tuple linguistic information. Expert Systems with Applications 2010(37),pp: 7895-7900.

[4] Wei GW , Grey relational analysis method for 2-tuple linguistic multiple attribute group decision making with incomplete weight information. Expert Systems with Applications 2011,38,pp: 4824-4828.

[5] Wei GW, Some generalized aggregating operators with linguistic information and their application to multiple attribute group decision making. Computers \& Industrial Engineering 2011,61,pp:32-38.

[6] Wei GW, Zhao XF, Some dependent aggregation operators with 2-tuple linguistic information and their application to multiple attribute group decision making. Expert Systems with Applications 2012,39,pp:58815886.

[7] Wan SP , 2-Tuple linguistic hybrid arithmetic aggregation operators and application to multi-attribute group decision making. Knowledge-Based Systems 2013(45),pp:31-40.

[8] Xu YJ, Wang HM, Approaches based on 2-tuple linguistic power aggregation operators for multiple attribute group decision making under linguistic environment. Applied Soft Computing 2011,11,pp:3988-3997.

[9] Li DY, Meng HJ, Shi XM, Membership clouds and membership cloud generators. Comput Res Dev 1995,32(6),pp:15-20. 
[10] Li LL, Liu LX, Yang CW, Li ZG, The comprehensive evaluation of smart distribution grid based on cloud model. Energy Procedia 2012,17,pp: 96-102.

[11] Niu DX, Fan LL, Technology feasibility evaluation of grid-connected distributed generation based on cloud model. Systems Engineering Procedia 2012,4,pp:305-313.

[12] Fan LJ, Ling YX, Liao LC, Li BX, An improved evaluation method based on cloud models for situation consistency within the battlefield of joint operations. Procedia Engineering 2012,29,pp:1590-1595.

[13] Yang XJ, Yan LL, Zeng L, How to handle uncertainties in AHP: The Cloud Delphi hierarchical analysis. Information Sciences 2013,222,pp:384-404.

[14] Kahneman D, Tversky A, Prospect theory: An analysis of decision under risk. Econometrica 1979,47,pp:263-292.

[15] Liu PD, Jin F, Zhang X, Research on the multi-attribute decision-making under risk with interval probability based on prospect theory and the uncertain linguistic variable. Knowledge-Based Systems 2011,24,pp:554-561.

[16] Renato A, Talles T, Combining prospect theory and fuzzy numbers to multi-attributes decision making. Expert Systems with Applications 2012,39,pp:11487-11493.
[17] Fan ZP, Zhang X, Chen FD, Multiple attribute decision making considering aspiration-levels: a method based on prospect theory. Computers \& Industrial Engineering 2013,65,pp: 341-350.

[18] Jiang YP, Fan ZP, Property analysis of the aggregation operators for 2tuple linguistic information. Control and Decision 2003,18(6),pp:754757.

[19] Zhang Y, Fan ZP, An approach to linguistic multiple attribute decisionmaking with linguistic information based on ELOWA operator. Systems Engineer 2006,24(12),pp:98-101.

[20] Dong YC, Xu YF, Li HY, Feng B, The OWA-based consensus operator under linguistic representation models using position indexes. European Journal of Operational Research 2010,203,pp: 455-463.

[21] Tversky A, Kahneman D, Advances in prospect theory: Cumulative representation of uncertainty, Journal of Risk and Uncertainty 1992,5,pp:297-323.

[22] Wang JQ, Zhang Z, Multi-attributes decision-making method with incomplete certain information based on intuitionistic fuzzy number. Control and Decision 2009,24(2),pp:226-230.

[23] Ghosh A, Pal NR, Das J, A fuzzy rule based approach to cloud cover estimation. Remote Sensing of Environment 2006,100(4),pp:531-549 